\title{
ON THE COMMUTATIVITY OF CERTAIN RINGS
}

\author{
ALEXANDRA FORSYTHE AND NEAL H. MCCOY
}

1. Introduction. In his fundamental paper $[5]^{1}$ on Boolean rings, Stone showed that a Boolean ring, that is, a ring $R$ with the property that $a^{2}=a$ for every element $a$ of $R$, is necessarily commutative. Recently, Kaplansky [3] announced that this result can be extended and that, under certain rather strong conditions on the positive integer $n$, a ring $R$ is commutative if $a^{n}=a$ for every element $a$ of $R$. Furthermore, Jacobson [2] has now shown that this is true without restriction on $n$. In fact, he has established the following more general result:

THEOREM 1 (JACOBSON). If for each element a of a ring $R$ there exists an integer $n(a)>1$, depending on $a$, such that $a^{n(a)}=a$, then $R$ is commutative.

A simple calculation [2, p. 702] shows that every element of a ring satisfying the hypothesis of this theorem has finite additive order. Thus if a division ring satisfies the hypothesis, its prime field is necessarily finite. Theorem 8 of [2] then furnishes a short and elegant proof of Theorem 1 for the case of division rings. The proof of the theorem can then be completed by obtaining a "reduction to division rings," that is, a proof that the theorem is true for all rings if it is true for all division rings. Jacobson accomplishes this by use of some rather deep results on algebraic algebras. The principal purpose of the present note is to present a simple reduction to division rings which was obtained independently by the present authors. This, coupled with Jacobson's proof of the result for division rings, furnishes a short and simple proof of Theorem 1.

We shall give, in $\S 3$, an entirely elementary proof of this theorem for the special case in which $R$ is of prime characteristic $p$ and $a^{p}=a$ for every element $a$ of $R$. Such a ring is a p-ring [4], which is perhaps the simplest and most natural generalization of a Boolean ring.

2. The reduction to division rings. We shall, in fact, treat a somewhat more general case which may be of some interest in itself.

We recall that, according to Birkhoff [1], a ring $R$ is subdirectly irreducible if the intersection of all nonzero two-sided ideals in $R$ is a nonzero ideal. The following fundamental theorem is essential for our purpose:

Received by the editors January 7, 1946.

${ }^{1}$ Numbers in brackets refer to the references cited at the end of the paper. 
THEOREM 2 (BIRKHOFf). Any ring is isomorphic to a subdirect sum of subdirectly irreducible rings.

We shall begin by proving the following lemma:

LEMMA 1. In a ring $S$ without nonzero nilpotent elements, every idempotent is in the center.

Let $e$ be an idempotent in the ring $S$, and $x$ an arbitrary element of $S$. Then

$$
(e x e-e x)^{2}=e x e x e-e x e x-e x e x e+e x e x=0 .
$$

Thus $e x e=e x$, and a similar calculation shows that also $e x e=x e$. Hence $e x=x e$, and $e$ is in the center.

LEMMA 2. If $S$ is a subdirectly irreducible ring without nonzero nilpotent elements, then the only idempotents in $S$ are the zero and the unit element in case $S$ has a unit element.

To prove this, let us assume that $e$ is an idempotent in $S$, neither 0 nor 1 , and show that $S$ is not subdirectly irreducible. By Lemma 1 , $e$ is in the center of $S$ and thus the set of all elements of the form $x-e x, x \in S$, is a two-sided ideal $\mathfrak{a}$ in $S$. Furthermore, $\mathfrak{a}$ is not (0) since otherwise $e$ would be the unit element, contrary to hypothesis. Let $\mathfrak{b}$ be the two-sided ideal in $S$ consisting of those elements of the form $e x, x \in S$. Then $\mathfrak{b} \neq(0)$ since it contains $e^{2}=e \neq 0$. Now $\mathfrak{a} \cap \mathfrak{b}=(0)$, for if $x-e x=e y$, multiplication by $e$ shows that $e y=0$. Hence $S$ can not be subdirectly irreducible.

A ring $R$ is a regular ring [6] if for each element $a$ of $R$ there exists an element $x$ such that

$$
a x a=a .
$$

We do not assume the existence of a unit element. From (1), it follows that $a x$ is idempotent. If $R$ has no nonzero nilpotent elements, Lemma 1 shows that $a x$ is in the center of $R$, and hence (1) may be written in the form $a^{2} x=a$. Conversely, if $a \neq 0$, the existence of an $x$ such that $a^{2} x=a$ certainly implies that $a$ is not nilpotent. We have thus established the following lemma:

LEMMA 3. A regular ring $R$ has no nonzero nilpotent elements if and only if for each element $a$ of $R$ there is an element $x$ such that $a^{2} x=a$.

We may now easily complete the proof of the following theorem which is the principal result of this note: 
THEOREM 3. A regular ring $R$ is isomorphic to a subdirect sum of division rings if and only if $R$ has no nonzero nilpotent elements.

The necessity of the condition is obvious. To prove the sufficiency, we consider a regular ring $R$ without nonzero nilpotents which, by Theorem 2 , is isomorphic to a subdirect sum of subdirectly irreducible rings $R_{i}$. Now each $R_{i}$ is a homomorphic image of $R$ and therefore a regular ring and, by use of Lemma 3, we see that no $R_{i}$ has nonzero nilpotent elements. Thus, by Lemma $2, R_{i}$ can have no idempotents other than the zero and the unit element. Now if $a_{i}$ is any nonzero element of $R_{i}$ there is an $x_{i}$ such that $a_{i} x_{i} a_{i}=a_{i}$. Thus $a_{i} x_{i} \neq 0$ and is idempotent. It follows that $a_{i} x_{i}$ is the unit element of $R_{i}$ and, by a similar argument, $x_{i} a_{i}$ is also seen to be the unit element. Since every nonzero element of $R_{i}$ has an inverse, $R_{i}$ is a division ring.

Theorem 3 clearly contains, as a special case, the desired reduction of Theorem 1. For a ring $R$ satisfying the condition of Theorem 1 is obviously regular and has no nonzero nilpotents. Hence such a ring is isomorphic to a subdirect sum of division rings each of which satisfies the condition of the theorem.

We are indebted to the referee for the following remark. If $a$ is any nonzero element of an algebraic algebra without nonzero nilpotent elements, the subalgebra generated by $a$ is of finite order and semi-simple. It is therefore a full direct sum of a finite number of fields and thus the equation $a x a=a$ has a solution. Such an algebra is therefore regular and we have another proof of the following result due to Jacobson [2, Corollary to Theorem 6]:

CoRollary. An algebraic algebra without nonzero nilpotent elements is isomorphic to a subdirect sum of division algebras.

3. A special case. We now let $R$ be a ring of prime characteristic $p$ with the further property that $a^{p}=a$ for every element $a$ of $R$, and shall give an elementary proof that $R$ is commutative. ${ }^{2}$

If $a$ and $b$ are any two elements of $R$, we must have

$$
\begin{aligned}
(a+b)^{p}= & a+b=a^{p}+\left[a^{p-1} b\right]+\left[a^{p-2} b^{2}\right]+\cdots \\
& +\left[a b^{p-1}\right]+b^{p}
\end{aligned}
$$

where

$$
\left[a^{p-1} b\right]=a^{p-1} b+a^{p-2} b a+\cdots+a b a^{p-2}+b a^{p-1},
$$

and, in general, $\left[a^{i} b^{j}\right]$ is a sum of products of elements $a$ and $b$, in

${ }^{2}$ Professor Jacobson has informed us that he has also obtained a simple proof in this case. 
each term of which $a$ appears $i$ times and $b$ appears $j$ times. Since $a^{p}=a, b^{p}=b$, we see from (2) that

$$
\left[a^{p-1} b\right]+\left[a^{p-2} b^{2}\right]+\cdots+\left[a b^{p-1}\right]=0 .
$$

Now (4) is true for all elements $a$ and $b$, hence we may replace $b$ in turn by $2 b, 3 b, \cdots,(p-1) b$, thus getting the following system of equations, the first of which is merely equation (4):

$$
\begin{aligned}
& {\left[a^{p-1} b\right]+\left[a^{p-2} b^{2}\right]+\cdots+\left[a b^{p-1}\right]=0,} \\
& 2\left[a^{p-1} b\right]+2^{2}\left[a^{p-2} b^{2}\right]+\cdots+2^{p-1}\left[a b^{p-1}\right]=0 \text {, } \\
& (p-1)\left[a^{p-1} b\right]+(p-1)^{2}\left[a^{p-2} b^{2}\right]+\cdots+(p-1)^{p-1}\left[a b^{p-1}\right]=0 .
\end{aligned}
$$

Now let $m$ denote the Vandermonde determinant $\left|i^{i}\right|(i, j=1,2, \cdots$, $p-1)$, and $m_{1}, m_{2}, \cdots, m_{p-1}$ the cofactors of the elements of the first column of $m$. If we multiply the equations (5) by $m_{1}, m_{2}, \cdots, m_{p-1}$, respectively, and add, we get $m\left[a^{p-1} b\right]=0$. Now $m$ is known to be a product of positive integers less than $p$, hence is prime to $p$; thus we must have $\left[a^{p-1} b\right]=0$. A simple calculation, using the explicit formula (3), and making use of $a^{p}=a$, shows that

$$
0=a\left[a^{p-1} b\right]-\left[a^{p-1} b\right] a=a b-b a .
$$

Since $a$ and $b$ are arbitrary elements of $R$, this proves that $R$ is commutative.

\section{REFERENCES}

1. Garrett Birkhoff, Subdirect unions in universal algebra, Bull. Amer. Math. Soc. vol. 50 (1944) pp. 764-768.

2. Nathan Jacobson, Structure theory for algebraic algebras of bounded degree, Ann. of Math. vol. 46 (1945) pp. 695-707.

3. Irving Kaplansky, The commutativity of generalized Boolean rings, Bull. Amer. Math. Soc. Abstract 51-1-9.

4. N. H. McCoy and Deane Montgomery, A representation of generalized Boolean rings, Duke Math. J. vol. 3 (1937) pp. 455-459.

5. M. H. Stone, The theory of representations for Boolean algebras, Trans. Amer. Math. Soc. vol. 40 (1936) pp. 37-111.

6. J. von Neumann, On regular rings, Proc. Nat. Acad. Sci. U.S.A. vol. 22 (1936) pp. 707-713.

Vassar College and

Smith College 